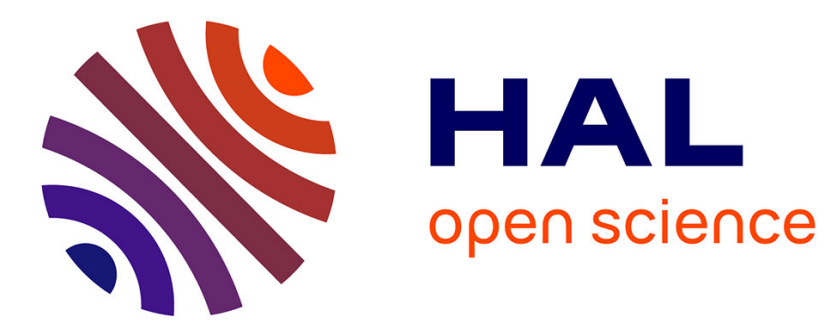

\title{
L'évaluation de la durabilité des contrats de plan État-Région, forme de management politico-administratif et vecteur de nouvelles pratiques d'aménagement
}

François Bertrand

\section{To cite this version:}

François Bertrand. L'évaluation de la durabilité des contrats de plan État-Région, forme de management politico-administratif et vecteur de nouvelles pratiques d'aménagement. Franck-Dominique Vivien, Jacques Lepart, Pascal Marty. L'évaluation de la durabilité, éditions Quae, pp.215-238, 2013, Collection Indisciplines, 9782759219049 10.3917/quae.vivie.2013.01.0215 halshs-01254549

\section{HAL Id: halshs-01254549 \\ https://shs.hal.science/halshs-01254549}

Submitted on 12 Jan 2016

HAL is a multi-disciplinary open access archive for the deposit and dissemination of scientific research documents, whether they are published or not. The documents may come from teaching and research institutions in France or abroad, or from public or private research centers.
L'archive ouverte pluridisciplinaire HAL, est destinée au dépôt et à la diffusion de documents scientifiques de niveau recherche, publiés ou non, émanant des établissements d'enseignement et de recherche français ou étrangers, des laboratoires publics ou privés. 


\title{
L'évaluation de la durabilité des contrats de plan Etat Région, forme de management politico-administratif et vecteur de nouvelles pratiques d'aménagement
}

\author{
François BERTRAND
}

Toute l'action publique contemporaine est désormais placée sous le signe du développement durable. Pour assurer cette orientation, l'évaluation des politiques publiques est un des outils largement mobilisé. Que produit la mise en œuvre généralisée de ces procédures d'évaluation de l'action publique au regard du développement durable ?

Ce texte propose d'observer ce point, en s'appuyant sur l'étude de l'évaluation de la planification régionale contractualisée, plus particulièrement l'étude des dispositifs régionaux d'évaluation stratégique mis en place à l'occasion de la préparation de la 4 ème génération des Contrats de Plan Etat-Région (CPER, portant sur la période 2000-2006) ${ }^{1}$, au niveau national et dans deux Régions françaises (Nord-Pas-de-Calais et MidiPyrénées) $)^{2}$.

Il est nécessaire dans un premier temps de rappeler le projet d'évaluation des politiques publiques, et sa diffusion au nom de l'amélioration et de la mise en cohérence de l'action publique.

Dans un second temps, les spécificités qu'introduit l'objectif du développement durable à ce projet d'évaluation seront soulignées. Il s'agit notamment de préciser ce que recouvre l'évaluation de la durabilité ( $v s$ évaluation environnementale et évaluation des politiques environnementales ou de développement durable), en quoi celle-ci s'avère méthodologiquement délicate sur plusieurs aspects (objectifs qualitatifs, enjeux multisectoriels, extension des horizons spatio-temporels, prise en compte des non-humains, etc.).

Enfin, l'observation des exercices régionaux au niveau national et plus précisément dans deux régions, doit permettre de clarifier les nombreuses fonctions remplies par l'évaluation dans les pratiques, en termes de pilotage de l'action publique et de déclinaisons régionales de la problématique du développement durable.

Pour finir, ce texte tentera de revenir sur les approches possibles et les intérêts relatifs en termes de recherche pour appréhender les activités évaluatives en tant que «technologies d'accompagnement du changement » (Rumpala, 2010).

\section{L'évaluation des politiques publiques}

Pour Meny et Theonig, «Évaluer une politique publique consiste dans le fait d'apprécier les effets attribuables à une intervention gouvernementale dans un domaine spécifique de la vie sociale et de l'environnement physique. »(Meny \& Thoenig, 1989 :

\footnotetext{
${ }^{1}$ C'est actuellement la $5^{\text {ème }}$ génération qui est en cours (2007-2013). Pour cette période, les Contrats de Plan Etat Région ont été renommés Contrat de Projet Etat Région.

${ }^{2}$ Cet article s'appuie sur un travail de doctorat, financé par l'ADEME (Bertrand, 2004). Il repose également sur une recherche-action (1998-1999) au sein du ministère de l'environnement (participation à la rédaction d'un guide méthodologique pour l'évaluation environnementale stratégique des CPER et des DOCUP 2000-2006) (Bertrand, 1999).
} 
289) L'activité d'évaluation comprend à la fois la production d'informations (relatives à la mise en œuvre, aux coûts, aux impacts, aux résultats, etc.) et leur interprétation afin d'émettre un jugement sur les effets de la politique. Cette activité nécessite donc une explicitation préalable des objectifs assignés à la politique, des valeurs au regard desquelles juger et un tiers évaluateur indépendant. L'évaluation entend donc «rendre compte » des effets de l'action publique. Ce faisant, elle peut également alimenter cette dernière en ressources de légitimité. "Non seulement l'évaluation est un effort qui vise à rendre compte de l'action des gouvernants mais elle participe bien d'un mouvement plus général de redéfinition des modes de justification du pouvoir politique dans les démocraties modernes (...). Il y a dans l'évaluation un double registre de connaissance et de justification. »(Duran, 1999 : 169)

\section{I.1. Les paradoxes de l'évaluation des politiques publiques}

Plusieurs paradoxes associés à l'idée d'évaluation des politiques publiques peuvent être rapidement soulignés. Le premier est certainement ce que Michel Conan appelle le paradoxe de la raison évaluative (Conan, 1996) : l'évaluation repose sur le doute de l'efficacité des solutions mises en œuvre en termes de politiques publiques pour répondre à un problème public et sur une profonde confiance dans cette même rationalité pour améliorer l'action publique, par l'analyse de ses effets au moyen d'un outillage de mesure. La pratique de l'évaluation peut également soulever certaine contradictions aussi bien pour l'action des agents administratifs, qui peuvent y voir tout à la fois une remise en cause de leur efficacité et un renforcement de leur rôle, que pour celle du personnel politique, pour qui l'évaluation peut représenter tout à la fois une perte de souveraineté sur la décision démocratique par un renforcement du contrôle technocratique et une production de nouvelles ressources de légitimité pour l'action politique.

\section{I.2. Origines contemporaines}

Plusieurs racines peuvent être attribuées au développement moderne de l'évaluation des politiques publiques comme outil de pilotage de l'action collective. En France, son origine contemporaine remonte au développement de la Rationalisation des Choix Budgétaires (RCB) dans les années 1970, qui visait l'amélioration de la mise en œuvre des politiques publiques. Au niveau régional, l'implantation de sa pratique repose largement sur l'accompagnement des Contrats de Plan Etat-Région (CPER) et encore davantage sur la mise en œuvre des programmes européens, pour lesquels elle fut très tôt obligatoire.

De cette filiation, l'évaluation apparaît avant tout comme un outil technocratique, exerçant un contrôle descendant. Le développement de l'évaluation vient également répondre au besoin de restaurer une confiance des citoyens envers l'efficacité et la légitimité de l'action publique sérieusement érodée ${ }^{3}$.

En fait, c'est un puissant levier accompagnant les transformations de l'action publique (décentralisation, contractualisation, mode «projet »...) et s'imposant rapidement comme une modalité privilégiée pour la conception et le pilotage de nouvelles politiques pluriacteurs, notamment en aménagement du territoire où contractualisation et évaluation apparaissent bien comme deux modalités privilégiées pour la conception et le pilotage des

\footnotetext{
3 "La demande d'évaluation crô̂t en même temps que les doutes sur la légitimité des politiques publiques." (Muller, 1990 : 122)
} 
politiques d'aménagement à plusieurs. A ce titre, leurs études peuvent être particulièrement révélatrices des nouvelles façons de faire l'aménagement.

Cette généralisation de l'évaluation s'est traduite sur le plan scientifique par une abondante littérature depuis le début des années 1990 (Monnier, 1992 ; Perret, 1993 ; Conan, 1996 ; Duran, 1999 : 167-196) et un foisonnement d'initiatives scientifiques et institutionnelles ${ }^{4}$. Dans la pratique, l'évaluation s'est propagée dans l'ensemble de l'appareil public, selon deux missions complémentaires qui lui sont attribuées (Chanut, $2002: 7)$ :

- une fonction cognitive : l'évaluation est alors au sein de services d'études et de recherches et vise la production d'informations et de connaissances, quantitatives et qualitatives ;

- une fonction stratégique : l'évaluation, placée dans les services en charge de l'animation et du pilotage des services, s'inscrit dans des visées ouvertement managériales et opérationnelles puisqu'elle remplit non seulement une fonction de définition et de structuration des politiques mais qu'elle est aussi chargée d'en faciliter et d'en améliorer la mise en œuvre.

\section{I.3. L'évaluation des politiques publiques parée de toutes les vertus}

L'évaluation est largement présentée comme un outil de rationalisation au service de l'amélioration continue de l'action publique : elle amènerait la mesure et la transparence, améliorant ainsi l'efficacité et le pilotage des politiques (cf. par exemple figure 1). Cette image d'un «long fleuve tranquille » de l'évaluation ne correspond pas aux pratiques étudiées.

\section{Objectifs}

Pertinence

Moyens Efficience
Efficacité

Résultats

Figure 1 : Les champs de l'évaluation

Source : Ministère de l'environnement, 1997

Tout d'abord au niveau national, le constat est souvent réalisé à répétition d'une déconnexion entre évaluation et action, marquant une profonde séparation entre action et connaissance : «(...) l'évaluation n'est pas organisée en vue de l'action (...) les dispositifs sont finalement relativement peu accessibles aux responsables de l'action publique. Leur capacité d'essaimage est limitée. (...) l'évaluation est fondée sur un modèle dépassé de l'expertise, une vision finalement très positiviste de connaissance où l'évaluation apparaît comme l'ultime avatar du rêve comtien d'une république des savants. Il n'est guère étonnant que l'évaluation soit accusée de produire des savoirs peu praticables et des résultats qui arrivent trop tard. » (Chanut, 2002 : 19)

\footnotetext{
${ }^{4}$ Comme en témoignent les rapports annuels du Conseil Scientifique de l'Evaluation depuis 1992 et, plus récemment, les nombreuses activités de la Société Française de l'Evaluation (SFE) créée en 1999. Et au sein de l'appareil politique, l'idée fait aussi florès comme le prouvent les travaux de la Mission d'Evaluation et de Contrôle (MEC) rattachée à la commission des finances de l'Assemblée nationale (créée en 1998) et ceux du comité d'évaluation des politiques publiques, groupe d'études rattaché à la commission des finances du Sénat créé en 2000.
} 
Pour bien saisir les rôles réelles que remplit l'évaluation dans les pratiques, il faut évidemment se défaire de cette représentation de l'évaluation reposant sur un modèle de décision à rationalité unique et suivant une dynamique linéaire et séquentielle (sur laquelle repose les trois temps de l'évaluation en tant qu'outil d'aide à la décision -ex ante-, de suivi -in itinere-, et de contrôle -ex post). Ce modèle a largement était critiqué (Sfez, 1973): les décisions s'élaborent davantage dans un contexte de «multirationnalités » (c'est-à-dire à minima sur un arbitrage plus ou moins clair entre différentes rationalités en jeu) et d'incertitude relative, où l'accès aux données comme le capital cognitif de chacun est très variable, et avec des finalités multiples. Sfez définit ainsi la décision contemporaine: "récit toujours interprétable, multirationnel, dominé par la multifinalité, marqué par la reconnaissance de plusieurs buts possibles, simultanés, en rupture » (Sfez, 1984: 118). Il faut donc également se défaire d'une représentation où l'amélioration des choix collectifs reposerait seulement sur une mise à disposition d'informations de meilleure qualité (plus précise, plus variée, plus robuste, etc.) "Généralement, dans la recherche appliquée aux décisions publiques, les faits sont incertains, les chiffres contestés, les enjeux importants et les décisions urgentes. »(Ravetz et Funtowicz, $1991: 86$ )

\section{L'évaluation au regard du développement durable}

L'évaluation de politiques publiques au regard du développement durable (c'est-à-dire de la mesure de leurs contributions positives ou négatives à l'objectif de développement durable) ne se restreint pas à l'évaluation de politiques spécifiques de développement durable (du type Agenda 21 local par exemple). Si l'évaluation de politiques de développement durable (tels les Agendas 21 locaux) peut se rattacher plus facilement aux pratiques "classiques" d'évaluation des politiques publiques, avec des adaptations particulières concernant la nature et les objectifs mêmes de ces politiques; le passage à l'évaluation des politiques publiques au regard du développement durable ouvre par contre un champ beaucoup plus vaste et moins bien moins stabilisé :

- non seulement en multipliant les champs des effets à prendre en compte : impacts environnementaux, effets sociaux, coûts et résultats économiques, mais également en terme de participation et de gouvernance ;

- mais ce premier point lui seul serait insuffisant car il ne constitue qu'une juxtaposition d'évaluations distinctes sur des domaines séparés, la transversalité souhaitée au départ s'effaçant alors au profit d'une nouvelle sectorisation. La prise en compte du développement durable par l'évaluation implique également le croisement des différentes dimensions, c'est-à-dire la prise en compte des interactions entre les impacts de différentes natures.

«La question classique de l'évaluation des politiques publiques semblent largement reformulée dès lors qu'il s'agit d'évaluer "au regard du développement durable » dans la mesure où ce n'est pas telle ou telle politique qui est directement évaluée mais plutôt en quoi telle politique ou tel programme d'action publique intègre les enjeux portés par le développement durable. »(Goxe, $2010: 73)$

Si les données sont toujours imparfaites pour asseoir parfaitement l'action publique, cette situation se révèle encore plus flagrante en ce qui concerne la complexité des problèmes associés au développement durable, le paradigme de la décision dans l'incertitude et l'information imparfaite conduit au principe de précaution (gestion des marges d'incertitude permettant le choix) et nécessité de dépasser l'application de modèles rationnels ou de simples analyses multicritères «Il ne peut pas être question de dégager, 
de l'extérieur ou d'en haut, par une planification rationnelle, une adéquation correcte des ressources à court et long terme. » (Brodhag \& Davoine, 2001) Il s'agit alors sans doute davantage de chercher à ménager des marges de manœuvre pour le futur, d'identifier des irréversibilités, de préciser le champ des négociations dans le cadre d'une rationalité procédurale (Faucheux et al., 1993).

Par bien des aspects, le développement durable implique une complexification de la question de l'évaluation qui interroge la finalité même de l'action locale : transversalité, pluridisciplinarité, certes, mais également élargissement des préoccupations dans le temps (prise en compte des générations futures), et dans l'espace (du territoire local au « reste du monde »).

\section{II.1. Les origines de l'évaluation au regard du développement durable}

L'évaluation de la durabilité des politiques publiques apparaît donc comme un challenge récent, qui doit croiser et non simplement superposer au minimum des évaluations avec des volets économiques, sociaux et environnementaux. Si des estimations socio-économiques sont depuis quelque temps déjà associées à la conception de nombreux programmes publics, le souci environnemental apparaît plus récemment. La seule "balise" préexistante en la matière demeure l'instauration en France depuis bientôt trente ans d'une étude d'impact sur l'environnement pour certains projets d'envergure ${ }^{5}$, complété en 2004 par l'évaluation environnementale stratégique pour certains plans et programmes ${ }^{6}$. Mais les méthodologies ne sont pas directement transposables.

L'évaluation au regard du développement durable s'est largement développée sur la base d'injonctions réglementaires, notamment celles encadrant les exercices de planification contractualisée (CPER) et encore davantage celles liées à la programmation régionale des fonds structurels européens. Cette poussée de l'évaluation par le niveau européen peut s'expliquer par la place éloignée qu'il occupe dans le jeu de la planification régionale, souffrant de fait d'un déficit d'information que le développement de l'évaluation est sensé pouvoir combler (Peyrefitte, 1996).

Ce développement sera ensuite généralisée par des textes «méthodologiques » produit par l'Etat (notamment le «Cadre de référence pour les projets territoriaux de développement durable et Agendas $21 »^{7}$ ).

Évaluation et développement durable sont fréquemment associés, notamment dans les textes onusiens, la première devant permettre la mise en œuvre effective du second ${ }^{8}$. L'évaluation se retrouve donc au premier rang des outils mobilisés pour mener la "croisade" de la soutenabilité. "L'évaluation, qui peine à s'ancrer fortement dans les pratiques administratives en France depuis le tournant des années 1980-1990, se trouve

\footnotetext{
${ }^{5}$ Les Etudes d'Impact sur l'environnement -EIE- s'appliquent au niveau des projets (et non pas au niveau des politiques, plans et programmes). Elles sont en vigueur en France depuis 1976 et en Europe depuis 1985.

${ }^{6}$ La directive européenne $\mathrm{n}^{\circ} 2001 / 42 / \mathrm{CE}$ du 27 juin 2001 relative à l'évaluation des incidences de certains plans et programmes sur l'environnement, transposée en France par l'ordonnance n²004-489 du 3 juin 2004, instaure le principe d'une évaluation environnementale stratégique (Strategic Environmental Assessment -SEA-) pour certains plans et programmes

${ }^{7}$ Ce cadre de référence institué par la circulaire du 13 juillet 2006 par la ministre de l'écologie et du développement durable est consacré par la loi du 12 juillet 2010 portant engagement pour l'environnement, dite «loi grenelle 2 »(titre VI «Gouvernance », Chapitre V «projets territoriaux de développement durable », articles 252-254).

${ }^{8}$ Le principe $\mathrm{n}^{\circ} 17$ de la Déclaration de Rio sur l'environnement et le développement des Nations Unies (juin 1992) avance bien l'évaluation comme un outil permettant la mise en œuvre concrète du développement durable
} 
élevée au rang de "modalité nécessaire» d'une "action publique durable 》 par les différents textes de références définissant le champ et les principes du développement durable. »(Goxe, $2010: 73)$

\section{II.2. Des difficultés conceptuelles}

Puisque l'évaluation est un des principes reconnus pour mettre en œuvre des politiques locales de développement durable, les difficultés conceptuelles que rencontre l'évaluation des politiques publiques au regard du développement durable rejoignent assez logiquement les difficultés d'opérationnalisation et de mise en pratique de l'objectif de développement durable dans l'action publique locale (ouverture des horizons spatiotemporels, mise en œuvre de la transversalité, gestion de l'incertitude, participation et association de la population...).

Pour le dire succinctement, la problématique du développement durable permet de mettre en avant des questions pour l'aménagement que posent la crise écologique globale mais aussi plus largement la crise de la modernité. Il s'agit de penser l'action du territoire dans un horizon spatio-temporel élargi, en intégrant les interdépendances avec ses extérieurs (les territoires voisins, et de proche en proche, le «reste du monde») (Zuindeau, 1996) et les conséquences à venir de ses actions (cf. par exemple la grille figure 2).

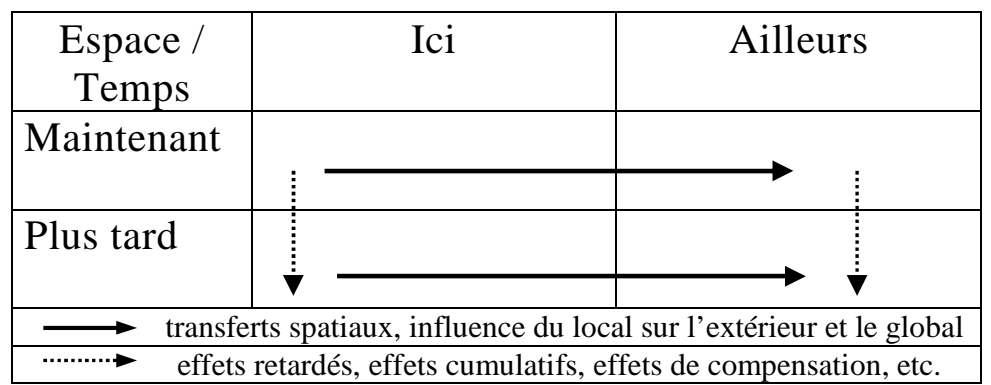

Figure 2 : Grille d'analyse spatio-temporelle pour l'évaluation des effets et des conséquences des pratiques agricoles

D'après J.-M. Legay (1993) cité par Landais (1998, p.11)

Les effets «ici et plus tard» et «ailleurs et plus tard»sont en partie imprévisibles et renvoient donc aux conséquences non intentionnelles des actions, ouvrant un questionnement plus large sur la possibilité d'une planification intégrant ces dimensions (Soubeyran \& Chalas, 2010).

D'un point de vue conceptuel, le défi est également d'élargir les processus décisionnels, représentant traditionnellement les intérêts des «acteurs forts » (les êtres humains, les générations actuelles, les pays du Nord), aux intérêts d'acteurs concernés et non-représentés, les acteurs "faibles" du développement durable, c'est à dire les territoires extérieurs, les générations futures ou encore les êtres vivants "non-humains" 9 . Force est de constater que les modalités, tant conceptuelles que pratiques, pour mettre tout le monde autour de la table, notamment les « acteurs faibles », restent largement à construire.

\footnotetext{
${ }^{9}$ Voir notamment les travaux de Bruno Latour en sociologie de la traduction sur la reconnaissance des nonhumains. Cf. notamment LATOUR B. (1999), Politiques de la nature. Comment faire entrer les sciences en démocratie, Éd. La Découverte, Paris, 383 p.
} 


\section{III. Évaluation, développement durable, CPER et DOCUP10}

Les réflexions qui suivent s'appuient sur les résultats d'un travail de doctorat qui a analysé l'exercice de conception de la planification régionale contractualisée (CPER et Document Unique de Programmation - DOCUP) sur la période 1998-2000, au niveau national dans un premier temps, puis plus précisément dans deux Régions menant des activités en matière de développement durable : Nord-Pas-de-Calais et Midi-Pyrénées (Bertrand, 2004). Une partie de ce travail s'est attachée à comprendre comment les dispositifs d'évaluation encadrant ces programmes étaient élaborés, de quelles façons ils étaient mobilisés dans le processus plus large de construction de ces programmes et à quels dispositifs de suivi ils aboutissaient.

Cette recherche n'a pas porté sur la mise en œuvre de politiques de planification et s'est limitée uniquement à leur conception, cependant l'analyse des dispositifs d'évaluation et de leur conception constitue un révélateur du sens et des objectifs attribués à ces politiques. L'évaluation des CPER fournit une approche transversale, "à cheval" entre action et réflexion, entre conception et mise en œuvre, mais aussi à cheval entre niveau socio-politique et niveau technico-scientifique.

\section{III.1. Les contrats de Plan entre l'Etat et les Régions, "révélateurs" du contenu et des pratiques de l'aménagement "à plusieurs"}

Les contrats de Plan entre l'Etat et les Régions sont des politiques multi-sectorielles, regroupant plusieurs éléments de politiques publiques. Un CPER est un document par lequel l'État et une Région s'engagent sur la programmation et le financement pluriannuels de projets d'importance (infrastructures, filières...). Conçu à l'origine comme un outil permettant d'assurer la cohérence entre le plan national et le plan de chaque région, les CPER ont perduré à la disparation de la planification nationale.

Les CPER représentent aujourd'hui une forme emblématique de la planification décentralisée, occupant une place particulière dans le paysage des politiques d'aménagement françaises : leur élaboration sur plusieurs années constitue à la fois un événement de la vie politico-administrative régionale et un levier important de modernisation des modalités d'intervention et de régulation de l'Etat. Ils marquent le développement des politiques contractuelles et l'avènement de la négociation institutionnalisée, demeurée longtemps en arrière plan, et souligne l'interdépendance entre les différents niveaux du système politico-administratif. L'évaluation des politiques, la définition de référentiels, d'indicateurs de développement durable, etc. font alors de plus en plus partie des outils de la négociation entre les différents niveaux institutionnels.

Pour ce qui concerne la préparation de la quatrième génération de CPER (dont la programmation s'étale de 2000 à 2006), le calendrier sera largement calqué sur celui des Fonds Structurels, dans une volonté légitime de coupler les deux exercices (CPER et DOCUP) qui partagent une certaine "communauté de pensées", notamment au niveau de la réflexion stratégique préalable qu'ils impliquent, des objectifs visés et de la diversité des domaines d'intervention qu'ils embrassent. Cette imbrication tant dans la pensée que dans la conception de ces politiques justifie d'ouvrir l'étude de la planification régionale contractualisée aux programmes européens (DOCUP), la préparation des DOCUP n'étant en effet guère dissociable dans l'analyse du processus d'élaboration des CPER.

\footnotetext{
${ }^{10}$ Document Unique de Programmation (programmes européens de développement régional au titre de Fonds structurels)
} 


\section{III.2. Le développement régional des dispositifs d'évaluation et l'imposition réglementaire de l'objectif de développement durable}

A la fin des années 1990, l'évaluation des politiques publiques s'est relativement bien diffusée en Régions, bien que variablement (nombreuses cellules d'évaluation créées, souvent rattachées aux directions stratégiques en charge de la planification et de la prospective) (Warin, 1999). En ce qui concerne l'évaluation environnementale, les situations régionales sont beaucoup plus contrastées (Bertrand 1999;2000; Bertrand \& Larrue, 2001). Et pour ce qui est de l'évaluation des politiques régionales au regard du développement durable, la période 1994-1999 n'a vu apparaître que de très rares tentatives embryonnaires visant à développer une méthodologie opérationnelle. Cependant, le développement des pratiques évaluatives en régions, quelles qu'elles soient, s'est largement effectué dans le cadre des CPER et des programmes européens (Lagant, 1998).

Le cadre réglementaire pour la contractualisation 2000-2006 ${ }^{11}$ instituait un renforcement des dimensions environnementales (meilleure connaissance de l'état de l'environnement local et anticipation, prévention, prise en compte des impacts potentiels sur l'environnement des stratégies de développement envisagées et des politiques associées) et plaçait l'objectif de développement durable comme une priorité générale chapeautant tout l'exercice de planification, dans un grand mouvement visant à en faire le plus grand dénominateur commun de l'action publique sans qu'un contenu réel soit précisé, au-delà d'une transversalité attendue, d'une réconciliation entre les dimensions économiques, sociales et écologiques et d'une concertation souhaitée.

Dans ce sens, les textes français insistent sur la nécessité d'identifier les besoins régionaux et d'apprécier l'intérêt de chaque projet à ses capacités de satisfaction des besoins économiques, sociaux, culturels et environnementaux. Au niveau de la Commission européenne, les quatre priorités qui se dégagent sont l'emploi, le développement durable, l'égalité des chances entre hommes et femmes et le développement des nouvelles technologies de l'information et de la communication (NTIC). Au niveau du gouvernement français, les trois priorités affirmées sont l'emploi, le développement durable et la cohésion sociale ${ }^{12}$. Au niveau de l'architecture et des exigences à fournir, le dispositif français, en proposant un processus idéal (Profil Environnemental Régional -PER-, évaluation ex ante et mise en place d'un tableau de bord avec des indicateurs de suivi) apparaît moins contraignant, davantage sur le ton de la suggestion que le dispositif européen.

\footnotetext{
11 Pour les CPER, l'ossature principale de ce cadre est formé par les circulaires du Premier ministre du 31 juillet 1998 «relative aux prochains CPER et aux principes relatifs à leurs architectures » et du 25 août 2000 « relative à la mise en cuvre de l'évaluation pour les procédures contractuelles pour la période 2000-2006», et par les circulaires de la ministre de l'Aménagement du Territoire et de l'Environnement aux préfets de région du $1^{\text {er }}$ juillet 1998 «relative à la préparation des prochains CPER», du 11 mai 1999 «relative aux prochains CPER », du 27 août 1999 « relative à la prise en compte de l'effet de serre dans les CPER».

Pour les DOCUP, c'est le règlement (CE) n 1260/1999 du Conseil du 21 juin 1999 «portant dispositions générales sur les Fonds structurels » (faisant suite à la proposition de règlement portant dispositions générales sur les Fonds structurels (COM(98) 131 final)).

${ }^{12}$ Notons que seuls les deux premiers objectifs d'emploi et de développement durable se retrouveront sans cesse affirmés. Pour le troisième, la cohésion sociale et territoriale, il semble moins fortement exprimé et pourra ainsi disparaître de temps en temps et être remplacé à certaines occasions par la participation, comme ce fut le cas par exemple dans l'éditorial du délégué à l'aménagement du territoire et à l'action régionale de la lettre de la Datar de l'automne 1999: «Les crédits en jeu sont importants, les objectifs sont novateurs: l'emploi, le développement durable, la participation. » Lettre de la Datar $\mathrm{n}^{\circ} 167$
} 
Au final, le développement durable est largement "affiché" sans que son contenu ne soit réellement explicité. L'analyse précise des textes indique que si la nécessité d'intégrer le développement durable aux contrats est largement affirmée, l'évaluation de cette intégration est essentiellement pensée sous ses formes environnementales ${ }^{13}$.

Une des principales conséquences de la promulgation du développement durable en tant qu'objectif prioritaire de la planification régionale est bien alors l'obligation d'évaluer les programmes au regard du développement durable. Le contexte juridique, en faisant du développement durable un référentiel formel, fournit une légitimité à cette problématique et participe à l'imposer comme un objectif central des stratégies de développement régional. Les pratiques évaluatives deviennent alors de véritables outils de la négociation entre les différents niveaux institutionnels. Elles participent à une ingénierie territoriale axée vers la définition de stratégies de développement partagées. Dans ce sens, l'évaluation peut apparaître comme un instrument de médiation politicotechnique permettant de matérialiser et de traduire techniquement les ambitions politiques des différents niveaux en objectifs pour l'action (objectifs de résultat).

Mais le renforcement des exigences en matière d'évaluation au regard du développement durable peut également recouvrir d'autres fonctions pour les niveaux supérieurs, et particulièrement pour l'échelon européen : développement d'un droit de regard et d'un droit à l'information (avec l'évaluation et la "contrainte" environnement) ainsi qu'un droit au refus légitime (au nom du principe de durabilité). Au niveau européen notamment, le développement de l'évaluation peut alors fournir un nouveau moyen de pression et un moyen d'affirmation sur les autres échelons de pouvoir.

\section{III.3. Principaux enseignements de l'évaluation préalable des CPER et DOCUP 2000-2006}

\section{Un calendrier serré, une faible anticipation et des "recyclages administratifs" entre procédures simultanées}

Les constats de ce paragraphe portent sur le volet environnemental des exercices d'évaluation, réglementairement largement plus encadré que pour le développement durable « en général ».

La période de préparation régionale des CPER et des DOCUP (1999-2000) correspond à un moment très chargé en termes de réflexions et de productions pour l'aménagement des territoires régionaux. Se déroulent simultanément à la préparation des CPER et des DOCUP, la préparation des Schémas de Services Collectifs (par les services de l'Etat en région) et des Schémas Régionaux d'Aménagement et de Développement du Territoire (SRADT - par les services de la Région), et cela dans un certain désordre par rapport à ce que prévoyait la Loi d'Orientation pour l'Aménagement et le Développement Durable du Territoire du 25 juin 1999 (LOADDT dite loi "Voynet").

Cette situation a permis tout au moins d'effectuer des "économies d'échelles" grâce à la réutilisation de documents déjà produits dans le cadre de procédures concomitantes,

\footnotetext{
13 Ainsi, au niveau national, la circulaire du 11 mai 1999, plus spécifiquement consacrée au développement durable, tente de préciser l'approche retenue par le ministère de l'Aménagement, notamment en termes de méthode, en fournissant des critères. Ces critères développent particulièrement les aspects environnementaux avec des indicateurs mieux précisés et placés à part des autres facteurs économiques et sociaux.
} 
même si ceux-là ne correspondaient parfois qu'imparfaitement ${ }^{14}$. Ainsi, même si le contenu des exercices demandés n'était pas identique, plusieurs régions ont "recyclé" les travaux des Schémas de Services Collectifs des Espaces Naturels et Ruraux (SSCENR), lorsqu'ils étaient suffisamment avancés, en tant que Profil Environnemental Régional (PER), ou du moins en tant que diagnostic environnemental régional minimum

Et ce mouvement de "recyclage administratif" a évidemment joué à plein entre la préparation en région du CPER et du DOCUP (essentiellement dans le sens contrat de Plan vers DOCUP mais pas seulement ${ }^{15}$ ). Il a pu également fonctionner pour servir à des documents à venir (SSCENR ou SRADT par exemple).

Malgré ces phénomènes, la fragilité et l'envergure limitée des dispositifs régionaux d'évaluation environnementale lors de la période 1994-1999 ont eu pour conséquence directe une intégration encore très restreinte de l'évaluation environnementale aux stratégies régionales de développement, par manque de moyens et de données disponibles à temps ${ }^{16}$. Cependant, avec une vision moins formelle, huit des dix-huit régions interrogées avaient constitué "à temps" -début 2000- un diagnostic environnemental, bien que parfois minimal et incomplet (Bertrand, $2004: 281$ ), ce qui, malgré tout, ne représente toujours pas la moitié de l'échantillon.

\section{Une déconnexion des temps de la décision et de l'évaluation mais des influences indirectes possibles : diffusion, sensibilisation, légitimation}

L'analyse des calendriers des négociations et du déroulement des exercices régionaux fait apparaître une déconnexion entre les temps de la décision et de la validation des programmes et les moments de réalisation de l'évaluation environnementale préalable (accusant jusqu'à un retard de plusieurs années). Cependant, ce constat ne saurait suffire. Car ce premier essai "grandeur nature" d'une évaluation environnementale a pu être l'occasion d'un ensemble de démarches, de collaborations et de pratiques nouvelles visant une meilleure prise en compte des préoccupations environnementales régionales et locales dans le choix des investissements de l'Etat, des Régions et de l'Europe. L'évaluation environnementale s'est donc à cette occasion matérialisée sous la forme de documents administratifs, sans que ceux-ci ne soient encore en mesure de peser directement sur les processus décisionnels. Les différents entretiens auprès d'acteurs soulignent également une influence indirecte en termes de sensibilisation des personnes ayant participé à ces démarches d'évaluation. Ce sont bien des fonctions pédagogiques ou endoformatives qui sont attribuées aux démarches d'évaluation, comme le soulignent également les travaux de Lascoumes et Setbon : «(...) l'apport de l'évaluation est plus sensible et plus profond au niveau administratif : comme moyen d'éclaircissement des problèmes, comme reflet élargi d'une action publique atomisée, comme lieu de confrontation des rationalités isolées, voire comme moyen d'amorcer une coopération toujours réclamée et rarement rencontrée. On peut affirmer que chez la plupart des administratifs impliqués dans l'évaluation

\footnotetext{
14 Cas de la Région PACA où les diagnostics environnementaux de deux Directives Territoriales d'Aménagement (DTA) en cours d'élaboration ont servi pour le contrat de plan, alors qu'ils ne couvraient qu'une partie du territoire régional.

${ }_{15}$ Ainsi, en Nord-Pas-de-Calais, la préparation du DOCUP Objectif $1 \mathrm{a}$ pu, en les précédant, alimenter certains travaux préparatoires au contrat de Plan.

${ }^{16}$ Trois PER seulement ont été réalisés dans des délais satisfaisants -début 2000- pour pouvoir jouer un rôle dans le processus d'élaboration des stratégies régionales -ce qui ne veut pas dire qu'ils en aient effectivement joué un(Bertrand, 2004: 281). Et ces trois régions étaient des régions pilotes retenues au niveau central pour l'expérimentation de cette première démarche d'évaluation environnementale préalable.
} 
domine une impression vivace d'apprentissage et celle d'une évolution relativement irréversible. » (Lascoumes \& Setbon, 1996 : 61-62)

Cette expérience d'évaluation environnementale a donné une visibilité accrue au niveau politique de problèmes et d'enjeux environnementaux dont la perception demeure floue, induisant une légitimation et une meilleure connaissance d'actions entreprises dans ce domaine. Elle a également permis des coopérations transversales interservices et interinstitutions (création de nouvelles scènes de dialogue). Il n'est cependant guère aisé de savoir si ces "passerelles" installées lors de la préparation des programmes se maintiennent par la suite, notamment en ce qui concerne les relations entre services (du Conseil Régional et de l'Etat en région).

Si une déconnexion entre les temps de la décision et ceux de l'évaluation est bien observée pour ce qui concerne l'évaluation environnementale, et par extension l'évaluation au regard du développement durable, celle-ci ne doit pas occulter l'importance des effets indirects du développement des pratiques évaluatives sur le système d'aménagement régional: visibilité accrue, légitimation des préoccupations environnementales et endo-formation du personnel chargé du suivi des programmes.

L'évaluation environnementale remplirait bien alors une fonction d'émergence, de construction et de légitimation de nouveaux problèmes (Bertrand, 1999). Le développement des procédures d'évaluation environnementale permettrait de renforcer l'acceptation de la mise en œuvre de politiques d'environnement, en légitimant leur nécessité (Lascoumes, 1994), notamment par des fonctions de sensibilisation des acteurs impliqués.

\section{Les principaux apports en termes de contenu}

Sur le contenu même des CPER et des DOCUP, cette expérience d'évaluation préalable aura permis d'établir un état initial ou état de référence en ce qui concerne la situation environnementale régionale (via l'établissement de Profil Environnemental Régional -PER-) et de doter ces programmes d'un cadre de référence, avec des objectifs de résultats et des indicateurs en matière de développement durable.

Cependant, les démarches d'évaluation au regard du développement durable étudiées n'ont pas abouti à caractériser clairement des formes souhaitables de durabilité locale. C'est-à-dire que le travail d'expression de frontières entre soutenable et insoutenable, collectivement définies par le personnel politique, en lien avec les populations concernées et appropriés aux territoires concernés, n'a pas été réalisée. A partir de cette carence, le report de ce travail de définition de seuils et de préférences sur les échelons techniques s'avère délicat et très limité.

Dans le détail, les aspects environnementaux bénéficient d'un effort d'ingénierie important pour mieux mesurer le poids écologique comme les gains en matière environnemental des programmes mis en œuvre, alors que les aspects sociaux comme les croisements socio-économique et socio-écologique demeurent beaucoup plus faiblement outillés.

\section{Les principaux apports en termes de processus}

L'évaluation apparaît davantage comme un processus d'apprentissage que comme un processus producteur de résultats concrets pour le pilotage des programmes (les résultats formels d'évaluation sont toujours relatifs, réduits et se périment vite). Pour les recherches 
effectuées, le processus apparaît plus riche par ses apports qualitatifs informels que par ses issues formelles.

Dans ce cadre, un des enjeux centraux apparaît bien être l'appropriation de systèmes d'évaluation co-construits et partagés par les différents acteurs concernés par les programmes $^{17}$.

L'organisation du dispositif d'évaluation est alors stratégique : certaines approches peuvent stimuler l'apprentissage en ouvrant des espaces de dialogue et d'ajustement, en permettant la rencontre entre acteurs de la politique à évaluer qui d'ordinaire ne se rencontraient pas, en permettant à ces acteurs d'avoir un regard réflexif sur leurs propres pratiques. «(...) il y a là en termes "d'organisation apprenante" des potentialités qui n'ont pas encore été exploitées pour l'évaluation. » (Chanut, $2002: 25$ )

L'évaluation stratégique au regard du développement durable peut alors être considérée comme un système de management catalyseur d'innovation institutionnelle et organisationnelle, permettant l'ouverture de "fenêtres d'opportunité", potentiellement en capacité de surmonter certains défis posés aux démarches territoriales de développement durable (transversalité, décloisonnement, acculturation, responsabilisation...). L'impact de ces travaux d'évaluation sur les décisions prises apparaît donc avant tout indirect, via des gains qualitatifs potentiels en termes de sensibilisation, endo-formation, interactivité, transversalité...

Il convient toutefois de ne pas "surévaluer" l'évaluation et ses apports aux dynamiques locales de développement durable. Ce n'est qu'un des outils d'apprentissage pour un processus d'apprentissage, au service des énergies et des volontés collectives, sans pouvoir mécanique propre. Elle ne peut à elle seule constituer une démarche territoriale de développement durable, encore moins surmonter les problèmes sur lesquels l'action politique se heurte.

\section{III.4. Enseignements des études régionales}

Sur la base des deux études régionales approfondies, en Midi-Pyrénées et en NordPas-de-Calais, en s'appuyant sur l'analyse de la genèse des dispositifs d'évaluation en région et l'étude fine de l'élaboration des dispositifs d'évaluation accompagnant la rédaction des CPER et des DOCUP pour la période 2000-2006, il est possible de préciser les formes et les modalités de ces démarches évaluatives, en distinguant les spécificités de chaque situation (cf. tableau 1), ainsi que de mettre en exergue certains des facteurs de «succès » et d'engagement communs à ces démarches. Faute de place, il n'est pas possible ici de détailler les exercices régionaux liés à l'évaluation, ni les dynamiques de traductions régionales du développement durable ${ }^{18}$. L'exemple du Nord-Pas-de-Calais est ici mobilisé à titre illustratif.

\footnotetext{
17 «L'enjeu de l'évaluation ne relève plus d'une normalisation des indicateurs, mais de la recherche d'une méthode partagée, visant à faire en sorte que les partenaires mobilisés définissent ensemble leurs objectifs, les impacts attendus et les critères permettant de vérifier régulièrement l'efficience de leur action et de procéder aux ajustements utiles (principe de l'évaluation endogène continue). »(Combe, $2003: 4$ )

${ }^{18}$ Pour de plus amples développement, on pourra se reporter à Bertrand, 2004 \& 2009.
} 
Tableau 1 : Comparaison des démarches régionales d'évaluation au regard du développement durable. Source : Bertrand, $2004: 391$

\begin{tabular}{|c|c|c|}
\hline & MIDI-PYRENEES & NORD-PAS-DE-CALAIS \\
\hline $\begin{array}{l}\text { Origine } \\
\text { "Epicentre" }\end{array}$ & $\begin{array}{l}\text { Dynamique plus technique } \\
\text { - Agence Régionale Pour } \\
\text { l'Environnement (ARPE) }\end{array}$ & $\begin{array}{l}\text { Impulsion politique } \\
\text { - Conseil Régional }\end{array}$ \\
\hline $\begin{array}{l}\text { Acteurs } \\
\text { pilotes }\end{array}$ & $\begin{array}{l}\text { - ARPE } \\
\text { - Collectivités locales pilotes } \\
\text { (Réseau des Villes Durables) } \\
\text { - Arrondissements pilotes } \\
\text { (D2MIP) }\end{array}$ & $\begin{array}{l}\text { - Conseil Régional (DPE) } \\
\text { - Etat en région (SGAR \& DIREN) }\end{array}$ \\
\hline $\begin{array}{l}\text { Principaux } \\
\text { partenaires }\end{array}$ & $\begin{array}{l}\text { - Bureaux d'Etudes } \\
\text { - Commission européenne }\end{array}$ & $\begin{array}{l}\text { - Bureaux d'Etudes } \\
\text { - Services régionaux }\end{array}$ \\
\hline $\begin{array}{l}\text { Principales } \\
\text { politiques } \\
\text { concernées }\end{array}$ & $\begin{array}{l}\text { - Politiques municipales } \\
\text { - Pays } \\
\text { - Parcs Naturels Régionaux } \\
\text { - Politiques expérimentales } \\
\text { (PACTE) et DOCUP (D2MIP) }\end{array}$ & $\begin{array}{l}\text { - Contrat de Plan Etat-Région } \\
\text { - Programmes européens (DOCUP) } \\
\text { - Politiques du Conseil Régional }\end{array}$ \\
\hline
\end{tabular}

Abréviations utilisées : DPE : Direction du Plan et de l'Evaluation (Conseil régional Nord-Pas-de-Calais)

D2MIP : Développement Durable en Midi-Pyrénées (programmation pilote au titre des fonds structurels, fonds européens, 19981999)

PACTE : programme européen pour l'évaluation de politiques régionales au regard du développement durable (1996-1997)

SGAR : Secrétariat Général aux Affaires Régionales / DIREN : Direction Régionale de l'Environnement

\section{Aperçu de la situation en Nord-Pas-de-Calais}

En Nord-Pas-de-Calais, la démarche d'évaluation apparaît bien ancrée, développée dès le milieu des années 1990 au sein du Conseil Régional, dans un contexte politique particulier (présidence verte de la Région). Une activité importante accompagnera la préparation des CPER et des DOCUP, avec l'élaboration d'une grille de 30 critères $\mathrm{d}$ 'analyse des politiques régionales par rapport au développement durable ${ }^{19}$. Cette « grille d'analyse de la cohérence des politiques régionales avec le développement durable » permettra notamment d'analyser la représentativité du système d'évaluation mis en place pour le pilotage du contrat de Plan, en associant des indicateurs de développement durable (IDD) intégrés au CPER aux critères de développement durable de la grille auquel ils se rapportaient le mieux ${ }^{20}$. Il ressort de ce travail une surreprésentation de l'environnement (un tiers des IDD du CPER), la parité bénéficie d'une représentativité importante au sein du dispositif ${ }^{21}$ alors que le champ de la participation et la gouvernance apparaît très faiblement abordé. Un quart des IDD couvre la satisfaction des besoins humains, tandis

\footnotetext{
19 Pour un aperçu de ces activités, cf. CONSEIL REGIONAL NORD-PAS-DE-CALAIS (2004), Le développement durable en question, Etudes Prospectives Régionales nº, mai 2004, 101 p.

${ }^{20}$ Travaux menés en interne en 2001 à la Direction du Plan et de l'Evaluation de la Région Nord-Pas-de-Calais.

${ }^{21} \mathrm{Au}$ point de constituer un champ à part entière, alors que ce thème ne correspond initialement qu'à un seul critère de développement durable de la grille du Conseil Régional, rangé dans la dimension «Satisfaction des besoins humains ».
} 
que le développement économique et la parité rassemble chacun environ un sixième des IDD.

Concernant la construction du système d'évaluation ex ante des DOCUP, les allersretours entre les différents niveaux et l'imposition par Bruxelles de ses champs d'intervention privilégiés laissent apparaître une certaine restriction des champs du développement durable au fur et à mesure de l'avancement de la construction du système de suivi. Ce processus de traduction réductrice $e^{22}$ aboutit au final à ce que les indicateurs de développement durable du système d'évaluation du DOCUP ne portent plus que sur deux domaines: l'environnement et l'égalité des chances entre les hommes et les femmes $^{23}$.

L'émergence du développement durable dans la planification régionale semble alors bénéficier avant tout à des "secteurs" institutionnels jeunes (comme l'environnement) ou naissants (comme l'égalité des chances entre les hommes et les femmes), qui en tirent des gains de légitimité. Tout au long de sa déclinaison et sa formalisation, l'objectif de développement durable, en quête de visibilité et de matérialité, s'appuie sur ces champs d'interventions en émergence qui lui sont étroitement liés. Ce mouvement de "concrétisation" correspond aussi aux moments de la négociation: énoncé selon des principes généraux et généreux en amont du processus, le développement durable s'efface en tant que tel dans les négociations et les arbitrages politiques finaux. Cet effacement se réalise donc au profit de quelques domaines qui s'en trouvent renforcés sur le plan des négociations "techniques", c'est à dire pour la rédaction des mesures et des conditions de leurs mises en œuvre.

L'exemple du Nord-Pas-de-Calais montre que l'internalisation des systèmes d'évaluation ainsi que la longévité de réflexions et de pratiques approfondies permettent l'initiation de phénomènes d'acculturation au sein du personnel technique et, dans une moindre mesure, au sein de la classe politique.

En matière de décloisonnement intra-institutionnel, le Conseil Régional apparaît comme une organisation plus "légère" comparativement aux services de l'Etat en région, organisés sectoriellement en branches déconcentrées de l'Etat central. Mais à l'inverse, dans des contextes où une certaine réticence des élus vis-à-vis de l'évaluation perdure (celle-ci étant alors perçue comme un contrôle dérangeant, illégitime et dangereux), les services de l'Etat en région, par rapport à ceux de la Région, peuvent bénéficier d'une marge d'autonomie plus importante quant à l'installation de démarches évaluatives ${ }^{24}$.

\footnotetext{
${ }^{22}$ Une responsable de ce dossier au SGAR exprimait ainsi ce mouvement : «(...) c'est à dire que nous avons réduit la dimension développement durable à environnement et égalité des chances hommes femmes. Parce qu'on avait un fort impératif de réduction du nombre d'indicateurs, on a dit: "Au sein du développement durable, il y a 2 dimensions qui sont privilégiées par l'Union Européenne : c'est l'environnement et l'égalité des chances ". » Extrait d'un entretien avec un membre du SGAR, novembre 2000

${ }^{23}$ Le complément de programmation du DOCUP précise les conditions exactes de mise en œuvre et d'attributions des Fonds. Des indicateurs de développement durable se retrouvent au niveau des Mesures, dans un paragraphe précisant la «Prise en compte des préoccupations du développement durable et d'égalité des chances ». Le ou les indicateurs de développement durable choisis portent soit sur l'égalité des chances, soit sur l'environnement, soit sur les deux.

${ }^{24}$ Ainsi, par la proximité des élus, certains critères de choix et indicateurs de mesure n'ont pas été généralisés des DOCUP au CPER : les critères d'éco-conditionnalité intégrés aux DOCUP n'ont pas été élargis au contrat de Plan. De la même manière, les indicateurs de développement durable concernant les transports semblent avoir été mis "en ballottage" au sein de la Direction des transports, en partie sous la pression des élus.
} 
Les activités évaluatives conduites au niveau régional apparaissent comme un des principaux outils disponibles au service des sphères politico-administratives pour la mise en œuvre du développement durable. Elles constitueraient alors bien des lieux d'émergence majeurs d'une compréhension régionale collective de la durabilité.

Mais on peut également observer un renforcement réciproque entre évaluation et durabilité, comme c'est le cas en Nord-Pas-de-Calais où le développement de l'évaluation et la réflexion d'ensemble autour du développement durable ont été deux modalités mobilisées par les Verts, alors à la tête de l'exécutif régional, pour tenter en douceur de «faire de la politique autrement». L'utilisation de l'évaluation fournissant «un chemin détourné pour poser les bonnes questions ${ }^{25}$, notamment dans le domaine des activités économiques, sans pour autant heurter de front des valeurs et des logiques politiques ancrées, alors que parallèlement l'association de l'objectif de développement durable à l'évaluation permettait un ambitieux essor par l'expérimentation d'une approche globale et transversale. ${ }^{26}$

Dans les deux situations régionales observées, les expériences d'évaluation ont été les principales scènes effectives pour mener une réflexion sur la déclinaison et l'intégration du développement durable dans les programmes de planification. Le processus d'évaluation au regard du développement durable représente alors l'application la plus concrète des injonctions à rendre «plus durable » l'action publique, et fournit un support pour une tentative de formalisation progressive de cette notion.

Pour la période étudiée, c'est à la fois un moment d'expérimentation (explorations de nouveaux domaines, recherches méthodologiques, expérimentations et tests sur des politiques spécifiques) et de diffusion de nouvelles pratiques.

\section{Les facteurs de succès}

Il est possible de dégager plusieurs facteurs de «succès », conditionnant un bon fonctionnement des démarches évaluatives au regard du développement durable. Tout d'abord, l'implication des acteurs et des différents services instructeurs en charge du pilotage des politiques sont indispensables pour que la démarche puisse s'incarner et exister véritablement. Il n'est alors plus question d'un tiers objectif en charge de l'évaluation car il n'est sans doute également plus question de rendre un jugement « objectif » sur la politique mais bien d'organiser au mieux sa construction et sa conduite par un processus d'implication améliorant son acceptabilité.

Le caractère ouvert du processus, sur les acteurs partenaires, sur les autres collectivités infra-régionales, sur les territoires extérieurs, conditionne logiquement la portée des démarches en termes d'émergence d'une culture commune et partagée.

Ensuite, la présence d'acteurs endossant la fonction d'agent de médiation, «à michemin entre savoir gestionnaire et art de la négociation » (Gaudin, 1999) apparaît nécessaire. Ce rôle peut être assuré en s'appuyant sur des consultants faisant office de "tiers médiateurs et animateurs". Enfin, un soutien politique, a minima un intérêt

\footnotetext{
${ }^{25}$ Extrait d'un entretien en mai 2001 avec Jean-François Caron, alors conseiller régional Vert et Vice Président du Conseil Régional Nord-Pas-de-Calais chargé de l'aménagement et du développement durable.

${ }^{26}$ "La démarche autour du développement durable a aussi été un "prétexte" pour asseoir un véritable processus d'évaluation, l'aboutissement d'une démarche évaluative et d'une démarche Contrat de Plan. (...) Le travail sur le développement durable a servi d'appui pour élargir la réflexion. » Extrait d'un entretien en novembre 2000 avec un membre de la Direction de la Planification et de l'Evaluation du Conseil Régional Nord-Pas-de-Calais.
} 
favorable, apparaît évidemment indispensable pour que la démarche ait une légitimité suffisante.

On le voit, le succès de ces démarches reposent beaucoup sur le facteur humain, d'où l'importance à accorder à la formation du personnel et au partage d'une «culture régionale » en termes de pratiques évaluatives comme de développement durable. Dans ce sens, le recrutement et la rotation du personnel peuvent également apparaître comme des points critiques pour la bonne conduite de ces démarches.

\section{La place des experts}

De la même manière, le rôle de passeurs de relais est crucial : constituant un tiers animateur par lequel un espace consensuel peut être dégagé, il remplit en quelque sorte le rôle "d'accoucheur" d'une compréhension partagée du développement durable. Sur ce point, des travaux sur la place et le rôle de l'expert seraient à mobiliser et à partager. Le développement durable offrant une "nouvelle idéologie professionnelle", en fournissant à de nombreux acteurs, experts, évaluateurs, administratifs et politiques une nouvelle légitimité d'action (opportunisme institutionnel et bénéfices tactiques), il y a un intérêt à initier et poursuivre des recherches sur la formation et les caractéristiques du champ en constitution des acteurs de la professionnalisation du développement durable (animateurs territoriaux, élus, consultants, universitaires...) ${ }^{27}$.

Le développement contemporain de ces dispositifs d'évaluation marque plus largement l'émergence d'un modèle de gouvernance où les sciences et techniques se voient attribuer le double rôle d'énoncer clairement l'impact des choix retenus et de légitimer l'action publique. "Face à l'effondrement de la confiance du citoyen dans les valeurs de la vie politique, elles [les sciences et techniques] deviendraient cet agent autonome capable par sa logique et sa démonstration, d'emporter l'adhésion de tous. » (Delaunay, 2001 : 75) En apportant plus de rationalité technique, mais aussi politique, les acteurs de l'évaluation, internes ou externes aux institutions régionales, participent à une nouvelle légitimation de l'action publique. Ces constations renvoient aux travaux déjà nombreux sur le rôle de l'expertise et la place des experts dans les processus décisionnels (Roqueplo, 1991 ; Callon \& Rip , 1991) et interroge également sur la place du chercheur dans ces dispositifs.

\section{L'introuvable public}

Enfin, sur la base de cette recherche, la mise en évaluation de l'action publique n'apparaît pas en mesure de modifier certains aspects des processus de construction «à plusieurs » des politiques d'aménagement du territoire. Bien que le partenariat soit indispensable et la concertation appelée des vœux de tous, l'ouverture au public de ces démarches demeure quasi-inexistante. Au niveau des évaluations des plans et programmes étudiés, la conception d'une "évaluation démocratique" ${ }^{28}$ reste largement utopique, rejoignant ici ces constatations de Daniel Béhar et Renaud Epstein issues du rapport du Conseil National de l'Évaluation de 2002: «La réalité de la pratique évaluative

\footnotetext{
${ }^{27} \mathrm{Cf}$. notamment sur ces aspects le colloque international «Instituer le développement durable » tenu à Lille les 8, 9 et 10 novembre, les travaux engagés dans le cadre de l'Action Concertée Incitative de recherche «Instituer le Développement Durable» [http://aciidd.net/] ainsi que l'ouvrage coordonné par Bruno Villalba, «Appropriations du développement durable. Émergences, diffusions, traductions » aux Presses Universitaires du Septentrion (2009).

${ }_{28}$ Telle qu'exprimée à la fin des 1980 par Patrick Viveret par exemple. VIVERET P. (1989), L'évaluation des politiques et des actions publiques, Rapport au Premier ministre, La Documentation française, Paris, 92 p.
} 
développée dans les villes, départements et régions est bien différente. La publicité des travaux évaluatifs demeure l'exception, se limitant la plupart du temps au seul cercle des acteurs concernés par la politique évaluée. Au mieux, les résultats évaluatifs font l'objet d'une publication administrative ou sont -très partiellement- repris par la presse régionale et locale. Au pire, l'évaluation se limite à un rapport qui pourra éventuellement servir à la mémoire administrative. » (Béhar, Boloquy \& Epstein, 2003 : 10)

La sous-représentation manifeste de la dimension participation et gouvernance dans les dispositifs peut s'expliquer d'une part par la complexité et la lisibilité réduite de programmes comme les CPER, et d'autre part, par une absence relative de savoir-faire en la matière.

Ce constat peut également être nuancé en soulignant que les dispositifs d'évaluation au regard du développement durable peuvent viser différents finalités qui oscillent, notamment suivant le niveau d'agrégation retenu, entre :

- une amélioration interne des systèmes de conception et de pilotage des politiques, sur un registre majoritairement technique (élaboration de batteries d'indicateurs et de critères constituant un «tableau de bord interne ») ;

- et une communication vers l'extérieur, dans un but de marketing territorial, avec des visées davantage politiques et démonstratives (indicateurs synthétiques de développement durable).

Les travaux d'évaluation sur lesquels s'appuient les analyses présentées ici relèvent très largement de la première catégorie.

Bien entendu, les frontières ne sont pas étanches entre ces deux types d'initiatives, qui sont bien au contraire le plus souvent étroitement imbriquées. Les démarches à visée essentiellement interne peuvent alimenter certaines actions de communication ; et symétriquement, les démarches cherchant plus à dégager une image des politiques menées à destination "de l'extérieur" ont bien évidemment des conséquences internes à l'institution concernée.

Idéalement, une double démarche pourrait être envisagée, couplant une diffusion en interne des résultats non pondérés des évaluations permettant l'appropriation de la démarche et de ses principaux enseignements par les acteurs, et une communication plus large des résultats synthétisés permettant d'effectuer l'indispensable retour vers la population et alimenter le débat et la participation des publics concernés par les politiques évaluées ${ }^{29}$.

\section{Les facteurs d'engagements}

Différents facteurs d'engagement à initier des démarches d'évaluation au regard du développement durable, outre les injonctions réglementaires des niveaux supérieurs, peuvent être identifiés et regroupés selon plusieurs ordres :

- pour des raisons d'ordre politique (intérêt et appropriation de cet enjeu par un élu ou un groupe d'élus) ;

- pour des raisons de conjonctures locales particulièrement difficiles : situations de crises locales écologiques (par exemple, effet de serre et pollution atmosphérique

\footnotetext{
${ }^{29}$ Un exemple de cette double démarche provient de la Région Nord-Pas-de-Calais où, après plus de cinq années d'activités sur ces questions d'évaluation des politiques régionales au regard du développement durable, une synthèse a été produite sous la forme d'une publication entièrement consacrée à ces questions (CR NPdC, 2004) afin de «dresser un état de ses travaux (...) pour en partager les enseignements et dessiner de nouvelles perspectives », comme le souligne la note accompagnant la publication.
} 
en Île de France, gestion de la ressource en eau en Bretagne...) sociales, économiques (régions en voie désertification par exemple) ou les trois à la fois (régions en reconversion industrielle par exemple comme le Nord-Pas-de-Calais) ;

- enfin, pour des raisons de marketing territorial, la communication autour du développement durable pouvant alors faire utilement office d'un label avantageux de qualité des territoires, sensée renforcer l'attractivité de ces derniers.

\section{En conclusion, quelle posture de recherche face à l'évaluation de la durabilité ?}

En matière d'évaluation de la durabilité, plusieurs approches sont possibles (cf. introduction de l'ouvrage) :

- Soit on postule que le développement durable est défini normativement par une série de critères objectifs. Une fois ceux-ci déterminés, l'exercice d'évaluation est organisé autour d'eux.

- Soit on considère que les contenus donnés au développement durable sont variables suivant les conditions spatio-temporelles, c'est-à-dire non fractals suivant les différents niveaux territoriaux, avec des contenus variant suivant les échelles considérées $^{30}$, mais aussi les époques. Ce sont alors essentiellement des critères subjectifs, à définir aux différentes échelles mais aussi suivant les différents contextes socioculturels. L'évaluation apparaît là davantage comme un processus de construction, d'expression et de confrontation des préférences de chacun afin d'établir une vision partagée.

Les travaux présentés ici montrent, à travers les dispositifs d'évaluation étudiés, une construction très procédurale du développement durable où, à partir d'un cadre réglementaire exprimant quelques grands principes généraux, sont élaborés les prémisses d'une compréhension régionale du développement durable, sur la base des conditions écologiques et socio-économiques du territoire, et en fonction des dynamiques et jeux d'acteurs en présence et des objectifs des politiques concernés. Un des intérêts de la diffusion du développement durable serait alors dans les instruments d'action publique construits et mobilisés en lui faisant référence (Lascoumes, 2005).

Il convient de préciser que ces résultats portent sur des exercices d'évaluation particuliers, très institutionnalisés, généralistes (la quasi-totalité des secteurs du développement régional sont concernés par les CPER et les DOCUP), multi-acteurs (associant un grand nombre d'acteurs) multi-niveaux (intervention de différents niveaux politico-administratifs) et marqués par une dimension technocratique importante.

Cependant, ces observations rejoignent des constations déjà effectuées de longue date : le flou conceptuel entourant la notion de développement durable conduit les acteurs en charge de sa concrétisation opérationnelle à s'appuyer sur les procédures et les méthodes afin de lui donner un contenu. La médiation technique permet alors «de construire un «contenu » opérationnel à ce concept» (Goxe, Holec \& Rousseau, 2006). Jacques Theys, qui a piloté le travail au sein de l'IFEN sur les indicateurs de développement durable à la fin des années 1990 ne dit pas autre chose à propos de la construction de ces indicateurs, «tentative pragmatique pour «donner une forme» à la problématique du développement durable», celle-ci apparaissant comme "un des rares

\footnotetext{
${ }^{30}$ Comme le rappelle Olivier Godard, la déclinaison territoriale du développement durable n'est pas fractale. «Selon les échelles territoriales considérées, les recommandations et les priorités ne seront pas les mêmes. » (Godard 1996: 34) Son contenu est redéfini suivant chaque échelle et ne répète pas les préoccupations de durabilité à l'échelle globale selon des contraintes territoriales homothétiques.
} 
moyens dont on dispose pour combler le fossé, précédemment évoqué, entre théorie et pratique dans le domaine du développement durable » (Theys, 2000)

Si cet apport procédural du développement durable est vérifié, la recherche sur les instruments mobilisés au service du développement durable doit également s'accompagner d'une réflexion politique sur le sens de ces outils (Villalba, Goxe \& Lipovac, 2005).

Ces situations semblent offrir un réel intérêt pour l'étude des formes territoriales de développement durable. Car si le développement durable «ne peut prendre corps historiquement qu'à travers l'action et la réflexion sur l'action »(Jollivet, 2001 : 115), les dispositifs locaux d'évaluation constituent bien des «dispositifs d'action » concrets (Jollivet, 2001) (de l'ordre tout à la fois de la technique, du règlement, de la procédure et de la négociation) qui permettent d'analyser la conception du développement durable à l'œuvre "à travers les priorités données à la maîtrise de tels ou tels processus ainsi que du choix de tel ou tel moyen pour atteindre les objectifs visés» (Jollivet, 2001 : 16).

L'évaluation du développement semble également fournir une nouvelle scène d'interaction entre acteurs, où s'expriment et se renouvèlent certaines aires de légitimité entre institutions, entre services et entre acteurs. Ces exercices d'évaluation peuvent donc également être considérés en tant que processus social d'apprentissage et d'acculturation. L'analyse de ces dispositifs d'évaluation peut éclairer la conception des politiques publiques, en renseignant précisément sur le processus de construction d'un référentiel territorial et des objectifs attribués aux politiques publiques. Cette position implique de comprendre l'évaluation non seulement comme un outil de management des politiques publiques mais également comme un outil de management des organisations publiques (Thoenig, 2002 : 40).

La nature spécifique de la problématique du développement durable (double nature entre idéal et réalité et double positionnement entre le monde scientifique et le monde de l'action) rend particulièrement délicat tout travail sur sa saisie par l'action collective. L'approche retenue ici conduit à l'aborder à la fois comme un objet et un outil de recherche:

- objet en tant que contenu renvoyant à des traductions et des appropriations localisées dans l'espace, mais aussi, à n'en pas douter, dans le temps (formes historico-géographiques),

- et outil en tant que cadre d'observation révélateur des processus à l'œuvre, donnant à voir la conception comme les formes de l'action publique.

\section{Bibliographie}

BEHAR D., BOLOQUY B. \& EPSTEIN R. (2003), «Évaluation et Pays : les enseignements du PaysBasque », 10 p. in CONSEIL NATIONAL DE L'EVALUATION (2003), Une évaluation à l'épreuve de son utilité sociale, rapport d'activités 2000-2002, La Documentation française, Paris, février 2003 [www.acadie-reflex.org]

BERTRAND F. (2000), "L'évaluation environnementale stratégique des programmes de planification régionale. Analyse du cadre réglementaire français et européens », pp. 127-147 In: SECRETARIAT FRANCOPHONE DE L'ASSOCIATION INTERNATIONALE POUR L'EVALUATION D'IMPACTS (2001), Les évaluations environnementales stratégiques, Actes du $5^{\text {ème }}$ colloque international des spécialistes francophones en évaluation d'impacts, 22-24 mai 2000, IAIA / AIEI - MATE, Paris, 1055 p.

BERTRAND F. \& LARRUE C. (2001), "L'environnement dans les dispositifs régionaux d'évaluation", pp. 256-280 In : BASLE M. \& GUIGNARD-HAMON C. (Ed.) (2001), Evaluation et gouvernance, Société Française d'Evaluation, Actes du $2^{\text {ème }}$ colloque de la SFE à Rennes, 15-16 juin 2000, SFE / 
Région Bretagne, Imprimerie de l'Université de Rennes 1, 617 p.

BERTRAND F. (1999), L'évaluation environnementale ex ante : outil d'aide à la décision et facteur d'intégration de l'environnement dans les pratiques et les concepts d'aménagement du territoire? L'exemple de l'évaluation environnementale stratégique pour la préparation des Contrats de Plan Etat-Région 2000-2006, mémoire de D.E.A., Maison des Sciences de la Ville, Université François Rabelais, Tours, 107 pp. + annexes

BERTRAND F. (2004), Planification et développement durable : vers de nouvelles pratiques d'aménagement régional ? L'exemple de deux Régions françaises, Nord-Pas-de-Calais et MidiPyrénées, Thèse de doctorat en Aménagement de l'espace et Urbanisme, sous la direction de Mme LARRUE, Université François Rabelais de Tours, 564 p. + annexes [manuscrit disponible en ligne : http://tel.ccsd.cnrs.fr/tel-00012142]

BERTRAND F. (2009), La traduction régionale de la problématique du développement durable. Analyse comparative des régions Nord-Pas-de-Calais et Midi-Pyrénées. pp. 187-212 In: VILLALBA B. (Ed.), Appropriations du développement durable. Émergences, diffusions, traductions, Presses Universitaires du Septentrion - Collection : Espaces Politiques, Lille, novembre 2009, 388 pages

BRODHAG C. \& DAVOINE P. (2001), «Evaluation, rationalité et développement durable », pp. 241-254 in BASLE M. \& GUIGNARD-HAMON C. (Ed.) (2001), Evaluation et gouvernance, Société Française d'Evaluation, Actes du colloque de Rennes de juin 2000, Imprimerie de l'Université de Rennes 1, 610 p. [www.agora21.org]

CALLON M. \& RIP A. (1991), «Forums hybrides et négociations des normes socio-techniques dans le domaine de l'environnement. La fin des experts et l'irrésistible ascension de l'expertise ", in THEYS J. (éd.) (1991/1992), Environnement, Science et Politique. Les experts sont formels, Actes du colloque d'Arc-et-Senans 1989, cahiers du GERMES, 3 vol., pp.227-238

CHANUT V. (2002), «L'évaluation : affaire d'Etat ou question d'organisation? », pp.1-33 In : Politiques et Management Public, vol. 20, nº 4, décembre 2002

COMBE H. (2003), «Evaluation des politiques publiques au regard du développement durable», communication au Séminaire Interdisciplinaire du Réseau Développement Durable et Territoires, 23 janvier 2003, Lille, $21 \mathrm{p}$.

CONAN M. (1996), L'évaluation constructive. Théorie, principes et éléments de méthodes, CSTB/Plan Urbain, Paris, $239 \mathrm{p}$.

DELAUNAY Q. (2001), "Evaluation et Gouvernance : innovation démocratique ou nouvelle rhétorique du politique », pp. 71-84 in BASLE M. \& GUIGNARD-HAMON C. (Ed.) (2001), Evaluation et gouvernance, Société Française d'Evaluation, Actes du colloque de Rennes de juin 2000, Imprimerie de l'Université de Rennes 1, $610 \mathrm{p}$.

DURAN P. (1999), Penser l'action publique, L.G.D.J., coll. droit et Société n²7, Paris, 212 p.

FAUCHEUX S., FROGER G. \& NOËL J.-F. (1993), "Quelle hypothèse de rationalité pour le développement soutenable », pp. 59-103 in Economie Appliquée, tome XLVI, 1993, nº

GAUDIN J.-P. (1999), Gouverner par contrat - l'action publique en question, Presses de sciences politiques, Paris, $233 \mathrm{p}$.

GODARD, O. (1996), "Le développement durable et le devenir des villes. Bonnes intentions et fausses idées », in Futuribles n²09, mai 1996, pp.29-35

GOXE A., HOLEC N. \& ROUSSEAU S. (2006) «Editorial dossier 8 : Méthodologies et pratiques territoriales de l'évaluation en matière de développement durable. », Développement durable et territoires [En ligne], mis en ligne le 14 décembre 2006, Consulté le 30 août 2010. URL: http://developpementdurable.revues.org/index3321.html

GOXE A. (2010), L'évaluation des politiques territoriales au regard du développement durable, pp.71-81 In : Développement durable et territoire (Zuindeau B., ed.), Presses universitaires du Septentrion, collection environnement et société, Lille, $517 \mathrm{p}$.

JOLLIVET M. (2001), «Introduction», pp.9-18 \& «Le développement durable, notion de recherche et catégorie pour l'action. Canevas pour une problématique hybride », pp.97-116 In : JOLLIVET M. 
(Ed.) (2001), Le développement durable, de l'utopie au concept, éd. Elsevier, coll. environnement NSS, Paris, 288 p.

LAGANT K. (1998), Analyse comparative des dispositifs régionaux d'évaluation et leurs travaux : la situation à fin février 1998 en France, Comité régional d'évaluation des politiques publiques, Préfecture de la région Bretagne, Région Bretagne, 119 p.

LANDAIS E. (1998), «Agriculture durable : les fondements d'un nouveau contrat social ? ", Le courrier de l’environnement de l’INRA n`33, avril 1998, pp.5-22 [www.inra.fr]

LASCOUMES P. (1994), L’éco-pouvoir, La Découverte, Paris, 317 p.

LASCOUMES P. \& SETBON M. (1996), L'évaluation pluraliste des politiques publiques. Enjeux, pratiques et produits, Groupe d'Analyse des Politiques Publiques - C.N.R.S., Commissariat Général du Plan, $182 \mathrm{p}$.

LASCOUMES P., 2005, «Le développement durable, vecteur d'innovations politiques ? », In : Smouts MC. (ed.), Le développement durable : les termes du débat, Paris, Armand Colin, pp. 95-108

MENY Y. \& THOENIG J.-C. (1989), Politiques publiques, coll. Thémis, PUF, 391p.

MONNIER E. (1992), Evaluation de l'action des pouvoirs publics, Economica, $2^{\text {ème }}$ éd. (1 ère éd. 1987), Paris, 245 p.

MULLER P. (1990), Les politiques publiques, PUF, Que sais-je ?, Paris, 128 p.

OFFNER J.-M. (2006), «L'indécision des lieux. Le surcode sfézien et l'action publique territoriale », in Alain Gras et Pierre Musso (dir.), Mélanges en hommage à Lucien Sfez, Paris, PUF, 2006, p. 143154.

PERRET B. (1993), «Réflexion sur les différents modèles d'évaluation », pp. 227-240 in Revue française d'administration publique $\mathrm{n}^{\circ} 66$

PEYREFITTE M. (1996), «Fédéralisme et politique régionale communautaire », XXXIIème colloque de l'ASRLF, Régions et villes dans l'Europe de l'an 2000, Berlin, 24 p.

RAVETZ J.R. \& FUNTOWICZ S.O. (1991), «Connaissance utile, ignorance utile, dissertation sur deux types de science », pp.83-94 in TYEYS J. (éd.) (1991), Environnement science et politique, Les experts sont formels, Actes du Colloque d'Arc et Senans de septembre 1989, éd. GERMES, Paris, $676 \mathrm{p}$.

ROQUEPLO P. (1991), «Introduction générale: l'expertise scientifique, convergence ou conflit de rationalités ? », in THEYS J. (éd.) (1991/1992), Environnement, Science et Politique. Les experts sont formels, Actes du colloque d'Arc-et-Senans 1989, cahiers du GERMES, 3 vol., pp.43-80

RUMPALA Y. (2009), «Mesurer le «développement durable» pour aider à le réaliser? La mise en indicateurs entre appareillage de connaissance et technologie d'accompagnement du changement », Histoire \& mesure 1/2009 (Vol. XXIV), p. 211-246.

SOUBEYRAN O. \& CHALAS Y. (2009), Incertitude, environnement, aménagement, quelle rupture?, In : Comment les acteurs s'arrangent avec l'incertitude ?, (Y. Chalas, C. Gilbert, D. Vinck (Dir.)), éditions des archives contemporaines, Paris, 135-157.

SFEZ L. (1973), Critique de la décision, Presses de la Fondation Nationale des Sciences Politiques, $4^{\text {ème }}$ édition, 1992, $574 \mathrm{p}$.

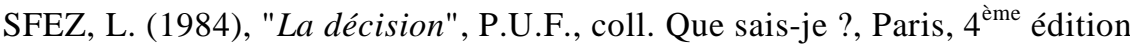

THEYS J. (2000), «Quelles recherches sur le développement durable? Un détour par les indicateurs », In : Développement durable Villes et Territoires - Du concept à sa mesure et à sa mise en œuvre : innover et décloisonner pour anticiper les ruptures, Notes du Centre de Prospective et de Veille Scientifique $\mathrm{n}^{\circ} 13$, MELT-DRAST, Paris, $135 \mathrm{p}$.

THOENIG J.-C. (2002), «L'évaluation en actes : leçons et perspectives», pp.33-50 in Politiques et Management Public, vol. 20, $\mathrm{n}^{\circ} 4$, décembre 2002

VILLALBA B., GOXE A. \& LIPOVAC J.-C. (2005), «Évaluer le développement durable: enjeux, méthodes, démarches d'acteurs», Développement durable et territoires [En ligne], Points de vue, mis en ligne le 15 décembre 2005, consulté le 20 décembre 2010. URL: http://developpementdurable.revues.org/1676 
WARIN Ph. (Dir.) (1999), L'évaluation en région, prisme d'une maturation politique et administrative, CERAT/CNRS - Institut d'Etudes Politiques de Grenoble, $232 \mathrm{p}$.

ZUINDEAU B. (1996), «La problématique du développement durable: les enseignements de l'approche spatiale », Colloque "Ecologie Société Economie", 23-25 mai 1996, Université de Versailles, 15 p. 
pp. 215-238 Vivien Franck-Dominique, Lepart Jacques, Marty Pascal, L'évaluation de la durabilité, Versailles Cedex, Editions Quæ «Indisciplines », 2013, 272 pages.

\section{AHHFA}

\section{L'évaluation de la durabilité}

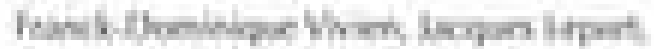

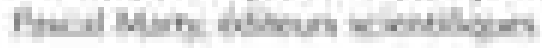

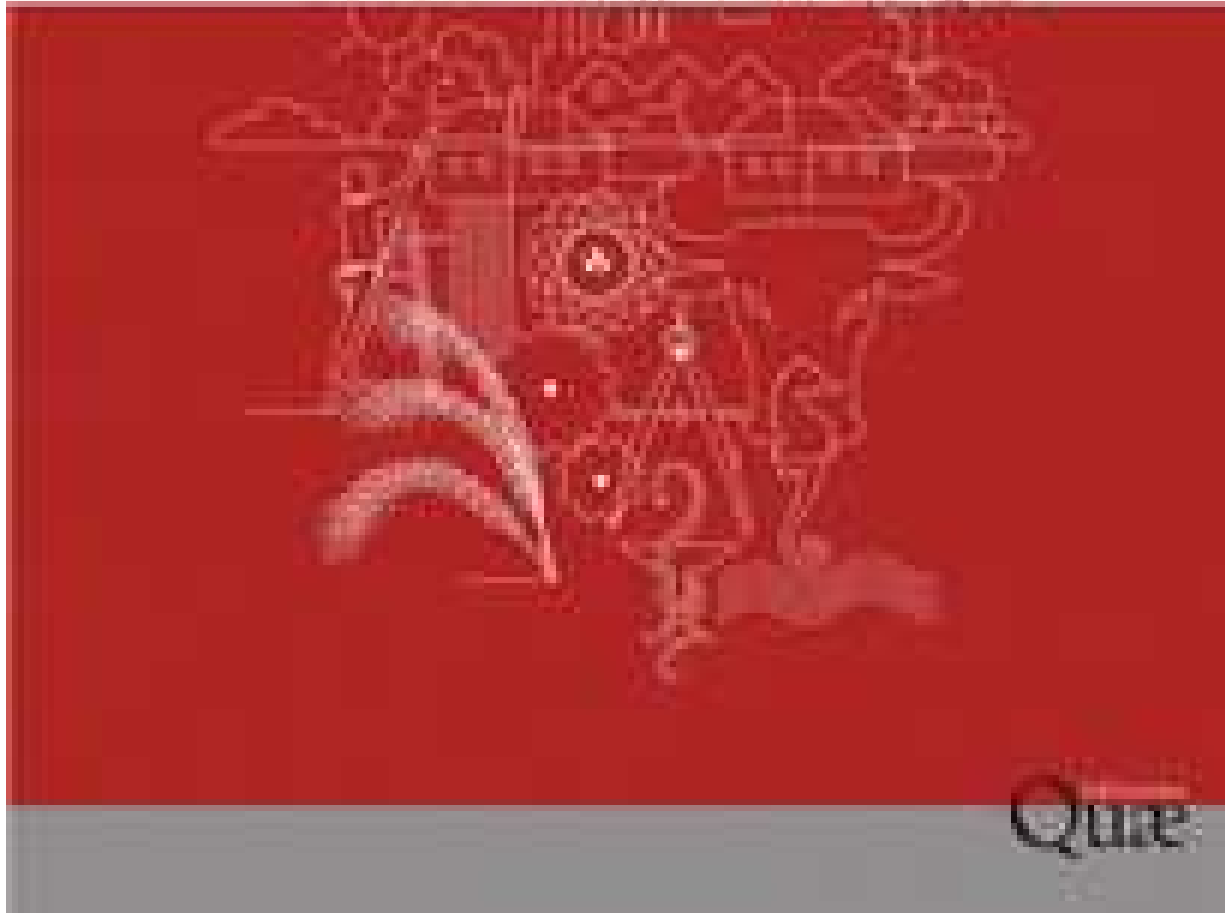

